\title{
Laboreal
}

Volume 14 N² | 2018

Digitalização e evolução do trabalho real

\section{Digitalización y evolución del trabajo real: introducción}

Digitalização e evolução do trabalho real: introdução

Digitalisation et évolution du travail réel: introduction

Digitalization and the evolution of real work: introduction

\section{Carole Baudin y Patricio Nusshold}

\section{(2) OpenEdition}

Journals

\section{Edición electrónica}

URL: http://journals.openedition.org/laboreal/583

DOI: $10.4000 /$ laboreal.583

ISSN: 1646-5237

\section{Editor}

Universidade do Porto

\section{Referencia electrónica}

Carole Baudin y Patricio Nusshold, « Digitalización y evolución del trabajo real: introducción », Laboreal [En línea], Volume 14 №2 | 2018, Publicado el 01 diciembre 2018, consultado el 25 septiembre 2020. URL : http://journals.openedition.org/laboreal/583 ; DOI : https://doi.org/10.4000/laboreal.583

Este documento fue generado automáticamente el 25 septiembre 2020

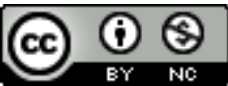

Laboreal está licenciado com uma Licença Creative Commons - Atribuição-NãoComercial 4.0 Internacional. 


\title{
Digitalización y evolución del trabajo real: introducción
}

\author{
Digitalização e evolução do trabalho real: introdução \\ Digitalisation et évolution du travail réel: introduction \\ Digitalization and the evolution of real work: introduction
}

Carole Baudin y Patricio Nusshold

\section{NOTA DEL EDITOR}

http://dx.doi.org/10.15667/laborealxiv218cbpn

1 Al escribir la convocatoria de este dossier temático, un debate se inició entre los miembros del comité. ¿Debemos hablar de ‘digitalización o de numerización’?

2 De hecho, los franceses, escépticos de apropiarse anglicismos poco utilizan el término de 'digitalización', cuando se usa en otros países francófonos o en castellano y portugués. Pero más allá del debate lingüístico y cultural, el uso (o no-uso) de este término es sintomático de problemas paradigmáticos.

El primero tiene que ver con la confusión de su significado. Como lo expone A. Mathé (2015), en francés, la diferencia semántica reside en que se hablará más de lo numérico (en oposición a lo analógico) para designar los soportes de la evolución tecnológica, su materialidad, mientras que lo digital y la digitalización serían términos más usados para describir las experiencias que provocan estas materialidades. Aunque en los discursos esta diferencia no es tan clara, esta primera reflexión muestra la complejidad del análisis que genera este fenómeno tecnológico al asimilar causas, medios y consecuencias, planteando también el problema de la focal con la cual se mira este fenómeno digital/numérico.

El segundo problema es que, muchas veces el uso del término 'digitalización' se hace en discursos insinuando una ruptura en la evolución tecnológica. Se anuncia una 'revolución' (de hecho, muchos hablan de cuarta revolución industrial). Desde un punto 
de vista histórico y antropológico (podemos citar los trabajos de Leroi-Gourhan, Mauss, Simondon, Lemonnier, Gras), la digitalización se enmarca en una dinámica evolutiva y continua del hombre para con las técnicas que crea. Sin embargo, la dinámica tecnológica que se vive actualmente, cuyo punto de inflexión se inició con la llegada de las Tecnologías de la Información y Comunicación (TIC) está dibujando una trayectoria exponencial tanto en su magnitud como en su rapidez. De hecho, algunos autores muestran lo continuo y disruptivo del fenómeno digital que se está dando (Valenduc \& Vendramin, 2016), ilustrando la complejidad de su lectura histórica.

5 En la literatura, la digitalización se ha considerado bajo diversas formas (automatización / robotización de la producción, surgimiento de la economía colaborativa, surgimiento de la mano de obra digital, trabajo a pedido a través de aplicaciones), y sus efectos se han analizado desde varias perspectivas: 1) a través de encuestas citadas a menudo como Frey \& Osborne, (2017) o el estudio de la Organización para la Cooperación y el Desarrollo Económicos (OCDE, 2017) sobre los riesgos de la automatización para ocupaciones y empleos. Asimismo, varios trabajos han estudiado los impactos de la economía digital en el mercado laboral, el empleo y la protección social (Degryse, 2016, 2017; Drahokoupil \& Fabo, 2016; Murillo, Buckland, \& Val, 2017; Valenduc \& Vendramin, 2016, 2017; Watson \& Stafford, 2016). 2) El giro digital también se estudia como un impacto en las formas de organización, gestión y liderazgo en las empresas (Loebbecke \& Picot, 2015). Correlativamente, la naturaleza, la estructura y el valor de las actividades laborales y las reacciones sociales e individuales relacionadas con ellas (tecnoestrés, hiperconectividad, hiperflexibilidad, precariedad social, fronteras borrosas entre la vida y el trabajo,) se han convertido en temas centrales en la literatura (Ayyagari, Grover \& Purvis, 2011; De Stefano, 2015; Shu, Tu \& Wang, 2011). 3) Finalmente, las transformaciones digitales se perciben como marcos legales desafiantes, en la legislación laboral (Prassl \& Risak, 2016), protección laboral o gestión del flujo de datos.

6 Este resumen bibliográfico muestra que estamos a la puerta de un cambio estructural drástico en el mundo del trabajo. No obstante, pocos son aún los estudios que relatan los cambios efectivos que provoca en las prácticas reales de trabajo. Si bien, desde los fines de 90', se han producido muchos conocimientos sobre la introducción de las TICs en el trabajo, sus efectos sobre las formas de trabajar, de interactuar, de construir nuevas prácticas, entre otros temas, el fenómeno que estamos viviendo sobrepasa los síntomas observados con la introducción de computadores o ciertas aplicaciones informáticas en el trabajo.

7 Se trata de algo más 'proteiforma' (Mériau \& Rousseau, 2017), puesto que, al abarcar transformaciones a distintos niveles y registros del trabajo, provoca efectos multidimensionales e interdependientes (complejos) que no son bien conocidos / y descritos.

8 La comunidad científica y experta en las condiciones de trabajo está iniciando un trabajo de recopilación de experiencias [1], generando así un corpus de conocimientos en el cual se quiere enmarcar este número.

9 En este cuadro, la propuesta de este dossier temático es, a través de una recolección de estudios realizados en distintas regiones y sectores, 1) observar cómo se aprende la dinámica digital en distintos contextos, 2) para contribuir la producción de conocimientos basado en la comprensión de su impacto en las experiencias laborales, y 3) para poner en debate los métodos de análisis e intervención que permiten optimizar las 'nuevas' condiciones de trabajo. 


\section{Focales sobre el fenómeno digital}

(1) de los transportes aéreos y los bancos. En sus análisis los autores muestran que esta ED abarca nuevos sistemas numéricos, pero también nuevas formas de organización en el trabajo. A través de un análisis sobre las modificaciones que provoca sobre los sistemas de trabajo, los autores proponen una reflexión sobre lo que implica estas transformaciones sobre la práctica de intervención ergonómica.

El tercer artículo de Elodie Chambonnière, Jacqueline Vacherand-Revel y Bruno Andrieu presenta los primeros resultados de un trabajo doctoral que analiza la transformación de la actividad laboral de una encargada en un gran grupo francés de la construcción con la integración de un nuevo sistema informático experto soportado sobre tableta táctil. Más allá del resultado, esta contribución propone una reflexión sobre los cuadros teóricos convocados para observar y analizar las implicancias del fenómeno digital en las dimensiones situadas y mediatizadas de las actividades laborales de este oficio productivo.

14 Finalmente, el cuarto artículo de Sacha Pinheiro, Marta Santos y Liliana Cunha describe las condiciones de implementación y los impactos del proyecto de digitalización llevado en el Instituto de Seguridad Social en Brasil. Este amplio estudio a nivel de escala y tiempo, permite a las autoras sacar conclusiones sobre las condiciones de implementación de la digitalización al comparar tres niveles de implementación y sus impactos sobre formas de apropiación de los cambios.

15 En todos los casos descritos, la digitalización consiste en la integración de soportes técnicos cuyo objetivo es optimizar el tratamiento, la gestión (análisis, resolución) y la difusión de informaciones. Cabe destacar que todos los autores muestran que estos nuevos soportes se sustentan en cambios organizacionales modificando los flujos de trabajo y los objetivos de productividad. Nos parece importante señalar que estas evoluciones no representan una reducción del trabajo humano sino por el contrario una transformación de la actividad, indispensable para poder encontrar soluciones creativas a los problemas para los cuales la tecnología es insuficiente.

La digitalización descrita no es solamente implementación de sistemas expertos. Se trata de la implementación de dispositivos multi-soportes, multi-modales, automáticos $\mathrm{y}$ artificialmente inteligentes, tratando $\mathrm{y}$ generando multitudes de datos/datas, controlados por algoritmos categorizando e interpretando fenómenos complejos para la toma de decisiones y la intervención en distintos niveles de las esferas del trabajo. 
17 Para entender las consecuencias y nuevas prácticas laborales, los autores del presente dossier miran el fenómeno digital en el trabajo desde un prisma comprehensivo, entendiéndolo como una construcción social desde un punto de vista antropotecnológico (Salini et al.) o con un enfoque instrumental del análisis de la actividad mediatizada (Leduc \& Ponge, Chambonnière et al., Pinheiro et al.), inspirado de los trabajos de autores como Rabardel (1995) para analizar los cambios en la interacción con los artefactos de trabajo, bajo un enfoque de 'clínica del uso' (BobillierChaumon \& Clot, 2016). Así, los objetos digitales del trabajo son vistos no solamente como una contingencia física en el trabajo sino a la vez como una contingencia sociotécnica, condición necesaria para entender el rol de estos nuevos no-humanos en el trabajo (Latour, 1991).

\section{Las contribuciones hacia un corpus teórico: impactos y nuevas prácticas}

18 Aunque la digitalización toma formas distintas, encuentra a menudo una configuración en los diversos casos descritos donde, los artefactos digitales se establecen como nuevas instancias de inteligibilidad (Sadin, 2015) y nuevas instancias de la corporalidad (Baudin, 2017a) en el trabajo real. Nuevas instancias en las cuales los trabajadores tienen poco margen de acción y apropiación.

19 Asimismo, las cuatro contribuciones exponen de qué manera los objetos digitales están modificando las prácticas laborales en 1) su materialidad espacial, temporal e interaccional 2) su organización individual, colectiva, social y funcional; y 3) sus representaciones individuales y colectivas.

20 Al proponer nuevos soportes virtuales, móviles y multimodales, para acompañar la actividad, se generan nuevas exigencias y cargas para los sujetos y los colectivos de trabajo. Por ejemplo, Leduc y Ponge muestran cómo la integración de soportes móviles en la actividad de los operadores de aeropuerto provoca un aumento de variabilidad de los objetivos y de los lugares de trabajo, obligando a las personas a actuar en forma permanente, en contextos dinámicos e instables. Salini et al., así como Pinheiro et al., evidencian los cambios de razonamiento que genera el soporte numérico en las actividades de oficina. La presentación de informaciones de distinto tipo, necesaria para tratar un problema, se cristaliza sobre una (o dos) pantalla(s), presentando las informaciones necesarias para la resolución en forma lineal y secuencial, obligando a los operadores a realizar manipulaciones permanentes para poder ver uno $\mathrm{u}$ otro documento electrónico, cuando antes los documentos impresos les permitían disponer de la información en forma global, heurística e inmediata. Sin hablar de las solicitaciones que esto genera a nivel de cargas visuales y posturales. Chambonnière et al., identifican las ventajas en poder presentar información sobre soportes móviles para el trabajo de directora de obra, pero señalan también como impiden o molestan actividades más informales que se suelen realizar en las obras para compensar atrasos. Finalmente, todos los casos analizando actividades de servicios revelan los cambios que estos nuevos soportes operan en las interacciones con los clientes ya sea porque reenvían la imagen de una mayor eficacia, elevando así el nivel de exigencia de los clientes o porque se oponen a las representaciones que los clientes tienen del oficio (como en caso de las enfermeras, Salini et al.). 
21 Al implementar sistemas informáticos codificados, e interconectados, los trabajadores se ven en la obligación de adaptarse a nuevas lógicas de categorización de sus actividades, generando nuevas cargas cognitivas, y solicitando nuevas competencias individuales y colectivas. Asimismo, Salini et al., muestran en qué medida las enfermeras se cuestionan sobre las formas de llenar la nueva historia clínica o 'dossier cliente', hasta donde brindar detalles, quien será el destinatario, etc. Plantea también el estatuto del 'saber profano' (Baudin, 2017b), este saber que los operadores construyen, a lo largo de su experiencia laboral. Un saber que pertenece al registro de lo sentido, de lo percibido, y que no tiene casillas en el programa informático.

22 Chambonnière et al., aunque describen poco el sistema en sí, aluden a las aptitudes mnémicas que supone por parte de la maestra de obra para retener los distintos proveedores/terceros y sus campos de intervención, información básica que debe introducir en el sistema para que sea útil. Estos autores muestran sobretodo la reconfiguración de los roles en el trabajo que supone. Asimismo, el sistema usado en la construcción al generar e-mails automáticos hacia los cuadros jerárquicos, establece nuevas formas de controles de calidad y productividad. Trazabilidad y control se fusionan. Los autores convocan a Foucault para ilustrar este riesgo.

23 El conjunto de los autores muestran de qué manera estos sistemas al rediseñar los circuitos de trabajo, al crear nuevas redes laborales, reconfiguran las reglas y la división del trabajo y a la vez desmaterializan (incluso desencarnan) el trabajo. En particular, todos dan a ver un rasgo común: la digitalización del trabajo se acompaña del aumento explícito o implícito de las exigencias de rentabilidad. Exigencias que no se pueden cumplir y que aceleran el ritmo de trabajo y la competición entre colegas. Pinheiro et al., Salini et al., y Leduc y Ponge ilustran, de hecho, el aumento de procedimientos que implican los objetos digitales que frenan y obstaculizan la fluidez de los procesos de trabajo e impiden los trabajadores no solamente alcanzar las metas organizacionales, sino que éstas se vuelven restricciones y carga de trabajo suplementarios. La aceleración del ritmo, la sobrecarga y la competencia entre quienes deberían estar cooperando opera en desmedro de la calidad del trabajo y del servicio ofrecido, tanto en términos de productividad como éticos.

Al desarrollar sistemas artificialmente inteligentes, basados en algoritmos y redes, que tratan y difunden en forma autónoma las informaciones, se está construyendo una forma de 'proletarización del saber' (Stiegler citado por Salini et al.). Los que eran hasta ahora los expertos de sus propios oficios, pierden el poder de la decisión y se vuelven a ser parte, o componente de un nuevo sistema de trabajo llevado por estos nuevos objetos. Estos sistemas inteligentes se fundamentan también sobre la noción de redes y aprendizaje. Muchos se basan en la captación, interpretación y extrapolación de los datos entrados por los mismos usuarios o clientes. Se construyen así nuevas formas de co-servicios. El cliente toma una parte de la actividad de construcción del servicio, pero quita así una parte de control del operador. Se externaliza de esta forma una parte del trabajo (Leduc \& Ponge, Pinheiro et al.). Los operadores se vuelven así un componente del sistema y muchas veces se ven obligados a la polivalencia para que el nuevo sistema de trabajo pueda funcionar. La polivalencia extrema da la sensación de una pérdida de pericia. Al disecar de esta forma el trabajo, los operadores pierden sus referencias, pierden el control de las informaciones, pero muchas veces se ven con nuevas formas de responsabilidades que no pueden asumir. 'El poder de actuar' está en juego en todos los casos. 
Lo que llama la atención a la lectura de estos estudios es que todos convergen en que las estrategias desarrolladas para trabajar con estos nuevos medios son principalmente estrategias de regulación o compensación muy exigentes para el trabajador, más que nuevos usos marcando cierta forma de apropiación. Se percibe una tendencia de adaptación de los trabajadores ya sea a nivel individual como colectivo para intentar responder a las nuevas configuraciones de trabajo, pero no se vislumbra todavía nuevas prácticas. Para comprender la no-apropiación de ciertas herramientas parece indispensable constatar la profundidad del cambio que se está operando en las experiencias laborales, que trastorna la significación, el sentido, el valor atribuido al trabajo, así como la cooperación no solo entre colegas sino también con los beneficiarios del servicio. Se perciben trabajadores cuyas nuevas actividades mediatizadas o digitalizadas, entran en conflicto con valores personales y colectivos.

La digitalización pone más que nunca, al centro de los debates, la brecha entre lo prescrito y lo efectivo, lo interpretado y lo real. Los artefactos digitales cristalizan en ellos la frontera material de este antagonismo fundamental de la ergonomía de la actividad, al ser la concretización de una representación prescrita del trabajo (desarrollada por los diseñadores y managers) y al poner en juego, en sus usos, la esencia misma del trabajo real para las personas.

\section{Reflexiones sobre las intervenciones en estas 'nuevas' condiciones de trabajo}

Con la digitalización son otros paradigmas que debemos convocar. No se trata solamente de una evolución de la numerización, en marcha desde la introducción de las TIC: con la digitalización se está definiendo una nueva configuración del trabajo real.

Asimismo, por ejemplo, los modos de implementación de esta digitalización plantean nuevas problemáticas. Con la excepción de Pinheiro et al., que focalizan su estudio sobre las condiciones de implementación del programa de digitalización del Instituto Nacional de Seguridad Social Brasilero, es interesante notar que los otros autores solo hablan de éstas condiciones en forma indirecta. Una de las explicaciones es la constatación que es necesario entender primero la digitalización y sus consecuencias para poder analizar las formas de implementarla. Estaríamos entonces en una etapa de inmadurez digital que impide tener las herramientas epistemológicas y teóricas adecuadas.

En las cuatro contribuciones, el fenómeno digital observado se desarrolla en un contexto de grandes empresas o grandes instituciones o corporaciones. En trasfondo están los 'abandonados' por la ola digital: trabajadores informales, precarios, probres, actividadas con bajo nivel de inversión. En las escalas estudiadas, se muestra un desarrollo digital siguiendo un camino 'top down', llevado con rapidez, en distintos ámbitos de la organización del trabajo.

En filigrana, se identifican dos estrategias de introducción e implementación distintas:

31 1) una estrategia 'brutal', acompañada de capacitaciones, que se realizan generalmente en paralelo de la introducción de los artefactos en las prácticas laborales (Leduc \& Ponge), a menudo tras su implementación.

2) una estrategia de desarrollo evolutivo de los instrumentos digitales, tanto de los soportes mismos - transferir soporte en tabletas sobre smartphones (Chambonnière et 
al.)- como de los programas informáticos (Salini et al.). o de las configuraciones organizacionales (Pinheiro et al.).

Ninguna de estas dos estrategias de implementación es adaptada según las conclusiones de los autores. Ya sea porque las capacitaciones son realizadas bajo un enfoque funcional (enseñar nuevas funciones con nuevos artefactos), o porque la voluntad de cambio gradual, o desarrollo evolutivo, -muchas veces sustentado por dinámicas de codiseño- trae con él, la necesidad de un ajuste permanente, un esfuerzo de actualización permanente de las prácticas que impide in fine a los trabajadores estabilizar sus entornos para que puedan ser habilitantes o actores de nuevas prácticas.

Con esta conclusión, Salini et al., proponen nuevos enfoques de formación para responder a los desafíos de la digitalización en los servicios estudiados. Proponen acompañar los procesos de apropiación con espacios de formación concebidos como espacios de intercambio de experiencias. Propuesta que se argumenta y se complementa con las de Pinheiro et al.

Leduc e Ponge así como Chambonnière et al, insisten en cambio en la necesidad de revisitar los cuadros analíticos, bajo la luz de estudios de casos, para poder intervenir en forma preventiva. Sus discusiones y conclusiones apuntan a que se debe acompañar el proceso de digitalización del trabajo a distintos niveles para poder preservar y optimizar las condiciones de trabajo. Ya sea interviniendo con los actores del management o con los diseñadores de las nuevas tecnologías.

\section{Conclusión}

La digitalización no es un 'mal necesario'. Se trata de una construcción social y humana. Puede existir solamente si el anclaje social existe. El fenómeno tecnológico que vivimos muestra que una parte de la sociedad está lista en acoger la ola digital. Sin embargo, este desarrollo tecnológico no solamente conlleva el riesgo de una fractura tecnológica o social, sino que desafía la noción misma de Trabajo para cada uno, para los investigadores y profesionales del Trabajo. Los sistemas informáticos no trabajan, sino que el trabajo humano evoluciona ante la confrontación con estas nuevas tecnologías.

La evolución de la digitalización pone en discusión los marcos teóricos y metodológicos con los cuales se debe captar, observar y analizar. Pone en discusión las formas de intervención que podemos proponer para reconocer el trabajo real realizado por los sujetos que se comprometen para realizar el trabajo lo mejor posible en estas 'nuevas' condiciones de trabajo.

Lo que nos enseña los cuatro artículos que componen este número temático es que la ola se está viviendo en forma similar en distintos contextos. Nos muestra también la necesidad de analizar esta evolución con enfoques comprensivos, analizando no solamente los artefactos digitales que la materializan, sino también las construcciones socio-técnicas que la materializan, y en las cuales se inscriben. Pasar por alto las dimensiones sociales y políticas en las cuales se inscriben estas evoluciones supondría dejar de lado en muchos casos las razones que justifican su implementación y las principales consecuencias negativas en términos de salud para quienes se encuentran confrontados con ciertas situaciones laborales. Finalmente, nos enseñan que como profesionales (científicos o expertos) de las condiciones de trabajo, es urgente que 
podamos intervenir sobre la construcción de estos artefactos para que sean reales 'acompañadores' de las prácticas laborales.

Para los ergónomos que trabajan con los diseñadores de estos artefactos, sabemos que el mayor riesgo se encuentra en la representación y la comprensión de los conceptos de trabajadores y de trabajo. Asimismo, se pueden escuchar muy a menudo discursos de managers o diseñadores que dicen 'reponer el humano al centro de las preocupaciones' o realizar 'procesos participativos' o 'co-diseño'. Sin embargo, en estos discursos o metodologías pre-hechas, el 'Humano' considerado es una figura codificada cuyas prácticas son desencarnadas para poder preservar las acciones útiles para la representación funcional del sistema de trabajo. Es importante que podamos reponer la complejidad y riqueza del trabajo humano, en sus dimensiones situada, social y encarnada, en el diseño de los nuevos espacios digitales de trabajo y acompañar las diferentes formas que toma la digitalización para que se puedan construir a partir del trabajo real y de la experiencia de las personas. Así, estas evoluciones no estarán al servicio de la aceleración del ritmo de trabajo, de la sobrecarga y la pérdida del sentido sino al servicio de la cooperación, de la salud, y de los resultados de los equipos de trabajo.

\section{BIBLIOGRAFÍA}

Ayyagari, R., Grover, V., \& Purvis, R. (2011). Technostress: technological antecedents and implications. MIS quarterly, 35(4), 831-858.

Baudin, C. (2017a). La perte des sens et du sens des corps technicisés. Actes FISO, Festival International de Sociologie, La fabrication des corps au 21e siècle.15p.

Baudin, C. (2017b). Au coeur du sensible: l'or 'profane' de Madre de Dios. In s. d. Ph.Geslin (Ed.), L'anthropotechnologie - Cultures et Conception (Ed. ISTE, pp. 71-101). London.

Bobillier-Chaumon, M-E., \& Clot Y. (2016). Clinique de l'usage : Les artefacts technologiques comme développement de l'activité, Revue @ctivités, vol. 13, numéro 2, 8p.

Bobillier-Chaumon, M-E., (2017). Du rôle des TIC dans la transformation digitale de l'activité et de la santé au travail, ANACT, la Revue des Conditions de Travail, Sept, nº, 16-24.

De Stefano, V. (2015). The Rise of the Just-in-Time Workforce: On-Demand Work, Crowdwork, and Labor Protection in the Gig-Economy. Comparative Labor Law \& Policy Journal, 37, 471-503.

Degryse, C. (2016). Digitalisation of the economy and its impact on labour markets. Research paper, ETUI, 81p.

Degryse, C. (2017). Shaping the world of work in the digital economy. ETUI, 11p.

Drahokoupil, J., \& Fabo, B. (2016). The platform economy and the disruption of the employment relationship. ETUI, 6p.

Frey, C. B., \& Osborne, M. A. (2017). The future of employment: how susceptible are jobs to computerisation? Technological Forecasting and Social Change, 114(January), 254-280. 
Latour, B. (1991). Nous n'avons jamais été modernes. Essai d'anthropologie symétrique, Paris: La Découverte.

Loebbecke, C., \& Picot, A. (2015). Reflections on societal and business model transformation arising from digitization and big data analytics: A research agenda. The Journal of Strategic Information Systems, 24(3), 149-157.

Mathé, A. (2015) Numérique ou Digital. In https://www.blogdumoderateur.com/numerique-oudigital/ [consultado en 14.03.2018]

Mériau \& Rousseau (2017). Mieux travailler à l'ère du numérique: Définir les enjeux et soutenir l'action, ANACT, la Revue des Conditions de Travail, Sept, $n^{\circ} 6$.

Murillo, D., Buckland, H., \& Val, E. (2017). When the sharing economy becomes neoliberalism on steroids: Unravelling the controversies. Technological Forecasting and Social Change, 125, 66-76. Organisation for Economic Co-operation and Development (OECD). (2017). OECD Digital Economy Outlook 2017. Paris: OECD.

Prassl, J., \& Risak, M. (2016). Uber, Taskrabbit, and Co.: Platforms as Employers-Rethinking the Legal Analysis of Crowdwork. Comparative Labor Law \& Policy Journal, 37(3), 604-619.

Rabardel, P. (2005). 13. Instrument, activité et développement du pouvoir d'agir. In Entre connaissance et organisation: l'activité collective (pp. 251-265). La Découverte.

Sadin, É. (2015). La vie algorithmique. Critique de la raison numérique. Paris: L'Échappée Shu, Q., Tu, Q., \& Wang, K. (2011). The impact of computer self-efficacy and technology dependence on computer-related technostress: A social cognitive theory perspective. International Journal of Human-Computer Interaction, 27(10), 923-939.

Valenduc, G., \& Vendramin, P. (2016). Work in the digital economy: sorting the old from the new. ETUI, 52p.

Valenduc, G., \& Vendramin, P. (2017). Digitalisation, between disruption and evolution. Transfer: European Review of Labour and Research, 23(2), 121-134.

\section{NOTAS}

1. Varias iniciativas se han organizado en estos últimos años. Para citar algunas realizadas en Francia últimamente están: la red ANACT-ARACT de Francia ha publicado en su Revista un número sobre numerización en el trabajo: “Mieux travailler à l'ère du numérique: Définir les enjeux et soutenir les Actions"; Revue sur les conditions de travail, n6, Spt. 2017; El CNAM realizó el 10 de Marzo del 2018, una jornada de reflexión sobre trabajo y numérico; la segunda edición de la universidad de verano multidisciplinaria e internacional del trabajo de Bordeaux (Francia) se desarrolló sobre el tema trabajo e innovaciones tecnológicas, entre el 2 y 6 de Julio 2018. 


\section{AUTORES}

\section{CAROLE BAUDIN}

Laboratoire 'User centered Design'

Haute École Arc Ingénierie

University of Applied Sciences and Arts of Western Switzerland, HES SO, Espace de l'Europe 11, 2000 Neuchâtel, Suisse

carole.baudin@he-arc.ch

\section{PATRICIO NUSSHOLD}

Laboratoire PCPP (Psychologie Clinique, Psychopathologie, Psychanalyse)

Université Paris Descartes - Sorbonne Paris Cité. Institut de Psychodynamique du Travail - ASTI, 7 rue Clovis, 75005, Paris, France

patricio.nusshold@ast-i.org 